\title{
Ephéméroptères du Sud-Ouest de la France. V. Premier inventaire des espèces recensées depuis 1870 (Insecta, Ephemeroptera)
}

\author{
A. Thomas ${ }^{1}$
}

Mots clés : Ephéméroptères, Sud-Ouest de la France, endémiques, espèces disparues.

Le Sud-Ouest de la France recouvre tout ou partie de 24 départements et totalise $128.000 \mathrm{~km}^{2}$. Une première liste de 60 espèces d'Ephéméroptères ne posant actuellement pas de problème de nomenclature ni de caractérisation est dressée. En fait, le Sud-Ouest de la France est la Terra typica de 10 de celles-ci : une décrite par Joly, deux par Eaton et sept par Thomas et al. Cinq sont des endémiques pyrénéennes, du seul versant Nord semble-t-il : Baetis catharus, B. nicolae, Rhithrogena kimminsi, R. strenua et Habroleptoides berthelemyi. En raison de leur appartenance à l'hyporhithron et à l'épipotamon, Rhithrogena germanica, Ecdyonurus macani et Ephemera vulgata, devenues très rares, sont gravement menacées par les pollutions d'origine agricole et la régulation des débits, en particulier les prélèvements d'eau pour l'irrigation, autorisés ou non, lors des étiages extrêmes. Pour les mêmes raisons, Isonychia ignota, Neoephemera maxima, Brachycercus harisella et Ephemera glaucops, non retrouvées depuis plus d'un siècle, ainsi que Prosopistoma foliaceum depuis plus de soixante ans, ont probablement disparu de cette région à l'heure actuelle.

\section{Mayflies of Southwestern France. V. First list of recorded species since 1870 (Insecta, Ephemeroptera)}

Keywords : Mayflies, Southwestern France, endemics, disappeared species.

Southwestern France consists of 24 departments and covers an area of $128,000 \mathrm{~km}^{2}$. A list of 60 unquestionably valid species of mayflies is provided for the region. The study area is the Terra typica of ten of the species : one described by Joly, two by Eaton, and seven by Thomas et al. Five species are endemic to the Pyrenees and are probably restricted to the north slope : Baetis catharus, B. nicōlāe, Rhithrogena kimminsi, R. strenua, and Habroleptoides berthelemyi. R. germanica, Ecdyonurus macani, and Ephemera vulgata are at risk because their hyporhithron and epipotamon habitats have been endangered by agriculture runoff, regulation, and diversion of the streams for irrigation, in particular when the water levels are already very low. For the same reasons, Isonychia ignota, Neoephemera maxima, Brachycercus harisella, and Ephemera glaucops have not been taken for over a century, and Prosopistoma foliaceum for more than 60 years, and all have presumably vanished from the region.

\section{Introduction}

Aucune synthèse n'a été effectuée sur la faune des Ephéméroptères du Sud-Ouest de là France. Le présent travail regroupe les citations d'Eaton sur cette région, ainsi que celles de quelques autres auteurs, essentielle-

\footnotetext{
1. Laboratoire d'Hydrobiologie, CESAC, UMR C 5576, Université Paul Sabatier, 118 route de Narbonne, F-31062 Toulouse Cedex, France.
}

ment E. Joly, Vayssière, Despax, Sowa, Müller-Liebenau, Thibault, Lavandier et Dumas, et Thomas et coll. Sauf exception, les résultats de Melle Verrier n'ont pas été repris ici, en raison de la grande incertitude des déterminations de cette dernière : au préalable, un examen détaillé de la collection Verrier est nécessaire.

En l'absence de la mention «leg.», il s'agit de mes propres récoltes (enrichies de la collection du regretté Claude Berthélemy), certaines déjà fort anciennes, exploitées ou pas dans deux manuscrits non publiés (Thomas 1970a, 1981), parfois même seulement citées comme matériel comparatif lors d'études d'autres es- 
pèces euroméditerranéennes. Au contraire, plusieurs mentions «leg.» sont relatives à des déterminations que j'ai effectuées dans le cadre de diverses thèses d'écologie des eaux douces, au cours des deux dernières décennies.

En ce qui concerne la famille des Baetidae, des concepts génériques récents (McCafferty et al. 1990, 1995 ; Waltz et al. 1994) ont été repris.

\section{Aire géographique considérée}

Le Sud-Ouest de la France est entendu ici au sens hydrologique du terme, c'est-à-dire incluant d'Ouest en Est les bassins versants de : la Charente, la Dordogne, la Garonne, l'Adour, le Tech, la Têt et l'Aude. Sur les côtes, les limites en sont l'embouchure de la Charente $\left(46^{\circ} \mathrm{N} / 1^{\circ} \mathrm{W}\right)$, près de Rochefort, et celle de l'Aude ( $\left.43^{\circ} 15^{\prime} \mathrm{N} / 3^{\circ} 15^{\prime} \mathrm{E}\right)$, entre Narbonne et Béziers.

Cette aire totalise $128.000 \mathrm{~km} 2$ et recouvre tout ou partie de 24 départements :

- en totalité : Ariège, Aude, Aveyron, Charente-Maritime, Dordogne, Haute-Garonne, Gers, Gironde, Landes, Lot, Lot \& Garonne, Pyrénées-Atlantiques, Hautes-Pyrénées, Pyrénées-Orientales, Tarn, Tarn-etGaronne ;

- pour leur plus grande partie : Cantal, Charente, Corrèze et Lozère ;

- pour une faible part (tout au plus 1/10ème de leur superficie) : Puy-de-Dôme, Deux-Sèvres, Vienne et Haute-Vienne.

L'amplitude altitudinale des prospections s'étend du niveau de la mer à une altitude de $2750 \mathrm{~m}$, dans les Pyrénées (Thomas 1975).

Les citations des départements prospectés sont présentées d'Ouest en Est (chaîne des Pyrénées) puis du Sud au Nord et sont abréviées ainsi : Ar. = Ariège ; Au. $=$ Aude $;$ Av. $=$ Aveyron $;$ Ca. $=$ Cantal $;$ C. - M. = Charente-Maritime ; H.-G. = Haute-Garonne $; \mathrm{Gi} .=\mathrm{Gi}-$ ronde $;$ Lot $=$ Lot $;$ L. \& G. $=$ Lot \& Garonne $;$ Loz. $=$ Lozère ; P.-A. = Pyrénées-Atlantiques $;$ H.-P. = HautesPyrénées $;$ P.-O. $=$ Pyrénées-Orientales $; \mathrm{T} .=$ Tarn $; \mathrm{T}$. $\&$ G. $=$ Tarn \& Garonne.

\section{Premier inventaire des espèces du Sud- Ouest de la France}

Famille des Siphlonuridae

Genre Siphlonurus Eaton, 1868

1. S. lacustris Eaton, 1870.

H.-P. : Vallée d'Aure : Grand lac de Port-Biehl $(2280 \mathrm{~m})$ et son déversoir ; Lac Long d'Estibère
$(2240 \mathrm{~m})$ et son déversoir ; Gourg de Nère Inférieur $(2200 \mathrm{~m})$; Lac d'Anglade $(2185 \mathrm{~m})$; Lac Inférieur d'Estibère $(2120 \mathrm{~m})$; Lac Vert $(2100 \mathrm{~m})$; Déversoir des Méandres d'Estibère (2040 m) ; Lac d'Aumar $(2190 \mathrm{~m})$; Laquettes $(2080 \mathrm{~m})$. Ainsi sont confirmées les citations anciennes de Despax (1927) et de Bertrand \& Verrier (1949a).

\section{Famille des Baetidae}

Genre Acentrella Bengtsson, 1912

2. A. sinaica Bogoescu, 1931.

P.-A. : Vinçon \& Thomas (1987). H.-G. : la Garonne à Portet $(145 \mathrm{~m})$, en amont de Toulouse. Au., Av. : Müller-Liebenau (1974).

Genre Alainites Waltz \& McCafferty, 1994

Ce genre a été érigé récemment (Waltz et al. 1994).

3. A. muticus (Linné, 1758).

P.-A. : Thibault (1971); Vinçon \& Thomas (1987). H.-P. : Thomas \& Thomas (1987). Au., Loz. : MüllerLiebenau (1974). Ca. : Sartori \& Thomas (1991).

Genre Baetis Leach, 1815

4. B. alpinus (Pictet, 1845).

P.-A. : Vinçon \& Thomas (1987). H.-P. : Thomas (1975). P.-O. : le torrent de la Coume des Fourats (2510, 2490 et $2450 \mathrm{~m}$ ), Massif du Carlit (Thomas 1981, 1995). Loz. : Müller-Liebenau (1974).

\section{B. buceratus Eaton, 1870.}

H.-G. : la Garonne à Toulouse (130 m) (Thomas et al. 1992).

6. B. catharus Thomas, 1986.

P.-A., H.-P. : Thomas (1986). H.-G. : le ruisseau de Surgeint, près d'Arbas (730 et 440 m). P.-O. : la Massane $(1000 \mathrm{~m})$ (J. Moubayed-Breil leg.).

7. B. fuscatus (Linné, 1761).

Ar. : l'Arize au Mas d'Azil (325 m). Au. : Müller-. Liebenau (1974).

8. B. gemellus Eaton, 1885.

P.-A. : Müller-Liebenau (1969, M. Thibault leg.) ; Thibault (1971) ; Vinçon \& Thomas (1987). H.-P. : Vallée d'Aure, la Neste de Géla (1440 m) ; la N. de Ba$\operatorname{det}(1400$ et $1350 \mathrm{~m})$; la N. d'Aragnouet $(1330 \mathrm{~m})$ (G. Gazagnes leg.). H.-G. : la Goute de Courbe près des Agudes (1395 m).

9. B. lutheri Müller-Liebenau, 1967.

H.-P. : le Ruisseau de Serres ( 830 et $820 \mathrm{~m}$ ) (G. Vinçon \& A.T. leg.). H.-G. : la Garonne en amont et en aval de St-Gaudens (365-350 m). Ar.: l'Arize au Mas d'Azil (325 m). Au., Av. : Müller-Liebenau (1974). 
10. B. melanonyx (Pictet, 1845).

P.-A. : Vinçon \& Thomas (1987). H.-P. : Thomas \& Thomas (1987).

11. B. nicolae Thomas \& Gazagnes, 1983.

H.-P., Ar., P.-O. : Thomas \& Gazagnes (1983).

12. B. rhodani (Pictet, 1845).

P.-A. : Müller-Liebenau (1969, M. Thibault leg.) ; Thibault (1971). H.-P., Au., Av., Loz. : Müller-Liebenau (1974). H.-G. : la Garonne en amont et en aval de Saint-Gaudens (365-350 m). Ar. : l'Arize au Mas d'Azil (325 m). P.-O. : la Massane (1000 m) (J. Moubayed-Breil leg.). Ca. : la Truyère à Chaliers $(740 \mathrm{~m})$ (A. T. \& C. Laur leg.).

13. B. scambus Eaton, 1870.

P.-A. : Thibault (1971). P.-O. : la Massane (1000 m) (J. Moubayed-Breil leg.).

14. B. vernus Curtis, 1834.

P.-A. : Vinçon \& Thomas (1987).

Genre Nigrobaetis Novikova \& Kluge, 1987

Ce genre a récemment été redéfini par Waltz et al. (1994).

15. N. digitatus (Bengtsson, 1912).

Au. : Müller-Liebenau (1974).

16. N. niger (Linné, 1761).

P.-A. : Thibault (1971). Ca. : le ruisseau de Monreysse à $680 \mathrm{~m}$, près d'Aurillac (G. Gazagnes leg.).

Genre Raptobaetopus Müller-Liebenau, 1978

17. R. tenellus (Albarda, 1878).

H.-G. : La Garonne à Portet (145 m), en amont de Toulouse (Thomas et al. 1992).

Genre Centroptilum Eaton, 1869

18. C. luteolum (Müller, 1776).

P.-A. : Thibault (1971), Vinçon \& Thomas (1987). H.-P. : Lavandier \& Dumas (1971). H.-G. : le Ruisseau de Cassignol (180 m) (C. Berthélemy leg.). Ar. : l'Arize au Mas d'Azil (325 m).

Genre Cloeon Leach, 1815

19. C. cognatum Stephens, 1835.

H.-G. : St-Hilaire $(200 \mathrm{~m})$; Toulouse $(130 \mathrm{~m})$; StLéon (270 m) ; St-Orens (180 m). Ar. : Moulis (430 m) (C. Berthélemy leg.). P.-O. : Fillols (C. Berthélemy leg.). C.-M. : Royan (10 m) (C. Berthélemy leg).

20. C. simile Eaton, 1870.

H.-P. : Vallée d'Aure : Grand Lac de Port-Biehl $(2280 \mathrm{~m})$ et son déversoir ; Lac Long d'Estibère $(2240 \mathrm{~m})$ et son déversoir ; Gourg de Nère Infé- rieur (2200 m) ; Lac d'Anglade (2185 m) ; Lac Inférieur d'Estibère (2120 m) ; Lac Vert $(2100 \mathrm{~m})$; Déversoir des Méandres d'Estibère (2040 m) ; Lac d'Aumar (2190 m) ; Laquettes (2080 m).

Genre Procloeon Bengtsson, 1915

21. P. bifidum (Bengtsson, 1912).

P.-A. : Eaton (1885), s. n. Cloeon rufulum Müller. Ar. : l'Arize au Mas d'Azil (325 m); le Volp à SteCroix (280 m) (C. Berthélemy leg.).

22. P. pulchrum (Eaton, 1885).

H.-G. : Eaton (1885), s. n. Centroptilum pulchrum Eaton.

\section{Famille des Oligoneuriidae}

\section{Genre Oligoneuriella Ulmer, 1924}

23. O. rhenana (Imhoff, 1852).

H.-G. : Eaton (1883), confirmant la citation d'E. Joly (1873) faite s. n. O. garumnica. Ar. : Eaton (1883). Au. : 1'Aude à St-Martin-Lys $(380 \mathrm{~m})$. Av. : le Lot à StGeniez (425 m) (A.T. \& C. Laur leg.). Loz. : le Lot à Montjézieu (535 m).

\section{Famille des Isonychiidae Genre Isonychia Eaton, 1871}

24. I. ignota (Walker, 1853).

H.-G. : Eaton (1883), s. n. Jolia roeselii (Joly), à la suite des citations très confuses d'E. Joly (1870 et 1872) ; Eaton (1885), s. n. Chirotonetes ignotus (Walker). A ma connaissance, l'espèce n'a pas été retrouvée depuis dans la région.

\section{Famille des Heptageniidae}

Genre Epeorus Eaton, 1881

25. E. torrentium Eaton, 1881.

Ar. : Eaton (1885) ; Berthélemy \& Thomas (1967). P.-A., H.-P., H.-G., P.-O., Loz. : Berthélemy \& Thomas (ibid.). Au. : l'Aude en aval de Puyvalador $(1370 \mathrm{~m})$. Av. : le Dourdou à Grand-Vabre (200 m) (A.T. \& C. Laur leg.). Ca. : la Truyère à Chaliers $(740 \mathrm{~m})$.

Genre Rhithrogena Eaton, 1881

26. $R$. germanica Eaton, 1885.

H.-G. : Despax (1949), s. n. R. haarupi Esben-Petersen ; Thomas et al. (1987). Ar. : Sowa (1971) (A. Thomas leg.).

27. R. kimminsi Thomas, 1970.

P.-A. : Vinçon \& Thomas (1987). H.-P., Ar. : Thomas (1970b). 
28. R. loyolaea Navas, 1922.

P.-A. : Vinçon \& Thomas (1987). H.-P. : Thomas (1970b, 1975). P.-O. : le torrent de la Coume des Fourats (2450 m), Massif du Carlit (Thomas 1981, 1995).

29. $R$. strenua Thomas, 1982.

P.-A. : Vinçon \& Thomas (1987). H.-P. : Thomas \& Gazagnes (1982).

Genre Ecdyonurus Eaton, 1868

30. E. angelieri Thomas, 1968.

P.-A., H.-P., Ar., P.-O. : Thomas (1968a). Au. : l'Aude, à $1 \mathrm{~km}$ en amont et à $4 \mathrm{~km}$ en aval d'Usson-lesBains (800 et $660 \mathrm{~m}$ ).

31. E. aurantiacus (Burmeister, 1839).

H.-G. : Eaton (1887), s. n. E. fluminum (Pictet) (pro parte) ; Thomas (1968a), s. n. pazsiczkyi Pongracz. Ar. : Eaton (ibid.), Thomas (ibid.). T. \& G. : Thomas (ibid.).

32. E. dispar (Curtis, 1834).

H.-G. : la Garonne à Toulouse (130 m). Ar., T., T. \& G. : Thomas (1968a). Av. : le Lot à St-Côme (345 m) et à Hauterive $(340 \mathrm{~m})$. Ca. : le Lot à St-Projet $(200 \mathrm{~m})$ (A.T. \& C. Laur leg.).

33. E. insignis (Eaton, 1870).

H.-G. : Eaton (1887) ; la Louge à St-Hilaire (200 m). Ar. : l'Arize au Mas d'Azil (325 m) ; le Volp de sa perte $(460 \mathrm{~m})$ au pont du Luquet $(240 \mathrm{~m})$. T. \& G. : la Garonne à Verdun-sur-Garonne $(105 \mathrm{~m})$. Av. : le Lot de St-Geniez (425 m) à la confluence avec le Dourdou $(190 \mathrm{~m})$ (A.T. \& C. Laur leg.). Loz. : le Lot à Montjézieu $(535 \mathrm{~m})$.

34. E. macani Thomas \& Sowa, 1970.

H.-G., Ar. : Thomas \& Sowa.(1970).

35. E. venosus (Fabricius, 1775).

P.-A. : Eaton (1887). H.-G. : la Garonne à Portet (145 m), en amont de Toulouse. Ar., Ca. : Thomas (1968a).

Genre Heptagenia Walsh, 1863

36. H. coerulans Rostock, 1878.

H.-G. : Eaton (1885), s. n. H. gallica Eaton; Thomas et al. (1992).

37. H. sulphurea (Müller, 1776).

H.-G. : la Garonne à St-Gaudens $(365 \mathrm{~m})$ et à Toulouse $(130 \mathrm{~m})$; la Louge à St-Hilaire $(200 \mathrm{~m})$. Ar. : l'Arize au Mas d'Azil (325 m).

\section{Famille des Ephemerellidae}

Genre Ephemerella Walsh, 1862

38. E. ignita (Poda, 1861).
P.-A. : Thibault (1971) ; Vinçon \& Thomas (1987). H.-P. : Lavandier \& Dumas (1971). H.-G. : la Garonne à Portet $(145 \mathrm{~m})$, en amont de Toulouse ; la Louge à StHilaire (200 m). Ar. : Bertrand \& Verrier (1950a et b) ; l'Arize au Mas d'Azil (325 m). Au. : l'Aude à St-Martin-Lys $(380 \mathrm{~m})$ et en amont de Carcassonne $(115 \mathrm{~m})$. P.-O. : Bertrand \& Verrier (1950a et b). Av.: Le Lot de St Geniez (425 m) à la confluence avec le Dourdou $(190 \mathrm{~m})$; la Truyère à Entraygues $(220 \mathrm{~m})$. Ca. : la Truyère à Chaliers $(740 \mathrm{~m})$. Loz. : le Lot du Bleymard $(1085 \mathrm{~m})$ à Montjézieu $(535 \mathrm{~m})$; la Truyère de La Villedieu (1225 m) au Soulier (865 m) (A.T. \& C. Laur leg.).

\section{Genre Torleya Lestage, 1917}

39. T. major (Klapalek, 1905).

P.-A. : Thibault (1971), s. n. T. belgica Lestage ; Vinçon \& Thomas (1987). H.-P. : la Neste d'Aure au pont de Bizous $(490 \mathrm{~m})$. H.-G. : le Nistos à Bonrepayre $(470 \mathrm{~m})$; la Garonne en amont de Saint-Gaudens $(365 \mathrm{~m})$. Au. : l'Aude en amont du pont de la D $32(1700 \mathrm{~m})$ et en aval de Puyvalador $(1370 \mathrm{~m}){ }^{-}$. Loz. : le Lot à Mende $(720 \mathrm{~m})$ et à Montjézieu (535 m) (A.T. \& C. Laur leg.).

\section{Famille des Neoephemeridae}

Genre Neoephemera Mac Dunnough, 1925

40. N. maxima (Joly, 1870).

H.-G. : E. Joly (1870), s. n. Caenis maxima, présence confirmée par Vayssière (1882), s. n. Tricorythus sp.? A ma connaissance, aucune nouvelle citation de cette espèce n'a été faite de la région.

\section{Famille des Caenidae}

Genre Brachycercus Curtis, 1834

41. B. harisella Curtis, 1834.

H.-G. : Eaton (1884).

La détermination spécifique (sur larve) par Eaton reste confuse (Sartori 1990), mais la détermination générique, elle, ne suscite aucun doute. Le genre étant monospécifique en Europe occidentale, de la Scandinavie à la Castille (Sartori, op. cit.), la citation de l'espèce est acceptée ici sans réserve, bien que cette dernière n'ait pas été retrouvée depuis dans la région, à ma connaissance.

Genre Caenis Stephens, 1835

42. C. beskidensis Sowa, 1973.

P.-A. : Thomas et al. 1986. H.-G. : La Garonne à StGaudens (365 m).

43. C. horaria (Linné, 1736).

H.-P. : Tabacchi (1987 : A. Thomas det.). Av. : le Lac de Pareloup (830 m) (J.N. Tourenq leg.). 
44. C. macrura Stephens, 1835.

H.-G. : Eaton (1884), s. n. C. halterata Eaton (= C. macrura : voir Malzacher 1986) ; la Louge à St-Hilaire (200 m). P.-A. : Thibault (1971).

45. C. pusilla Navas, 1913.

H.-G. : la:Garonne à Portet $(145 \mathrm{~m})$ et à Toulouse (130 m) (Thomas et al., 1992).

\section{Famille des:Prosopistomatidae}

Genre Prosopistoma Latreille, 1833

46. P. foliaceum (Fourcroy, 1785).

H.-G. : E. Joly (1871), N. Joly \& E. Joly (1872 \& $1875)$ et Vayssière (1881), toutes citations s. n. $P$. punctifrons Latreille. Gi. : Bertrand \& Verrier (1949b). Les dernières récoltes de larves de cette espèce dans les environs de Toulouse ont été, à ma connaissance, effectuées par Despax en 1929.

\section{Famille des Leptophlebiidae}

Genre Choroterpes Eaton, 1881

47. C. picteti (Eaton, 1870).

H.-G. : la Garonne à Portet (145 m), en amont de Toulouse. Ar. :Thomas \& Vitte (1988). T. \& G. : l'Aveyron à Nègrepelisse $(100 \mathrm{~m})$, en aval de la retenue (G. Gazagnes leg.).

\section{Genre Thraulus Eaton, 1881}

48. T. bellus Eaton, 1881.

H.-P. : Tabacchi (1987 : A. Thomas det.). P.-O. : Bertrand \& Verrier (1949a).

Genre Leptophlebia Westwood, 1840

49. L. vespertina (Linné, 1758).

Loz. : Lac de Saint-Andéol (Aubrac) (C. Berthélemy leg.).

Genre Paraleptophlebia Lestage, 1917

50. P. cincta (Retzius, 1783).

Ar. : Gagneur et al. (1985).

51. P. submarginata (Stephens, 1835).

P.-A. : Thibault (1971); Vinçon \& Thomas (1987). H.-P. : Lavandier \& Dumas (1971) ; le Canal de l'Ailhet près de Tarbes (305 m) (E. Tabacchi leg.). H.-G. : la Garonne en amont de St-Gaudens $(365 \mathrm{~m})$; le Tounis près de Cazères $(240 \mathrm{~m})$ (J. Y. Pujol leg.). Ar. : le Volp en amont de sa perte $(460 \mathrm{~m})$ et à Montardit $(430 \mathrm{~m})$ (C. Berthélémy leg.). Loz. : cascade de Dérols (Aubrac) (C. Berthélemy leg.).

Genre Habroleptoides Schoenemund, 1929

52. H. berthelemyi (Thomas, 1968).
P.-A. : Vinçon \& Thomas (1987). H.-P.,Ar., P.-O. : Thomas (1968b).

53. H. confusa Sartori \& Jacob, 1986.

P.-A. : Thibault (1971). Ar., P.-O., Ca., Loz. : Thomas (1968b). Toutes ces citations s. n. H. modesta (Hagen).

Genre Habrophlebia Eaton, 1881

54. H. lauta Eaton, 1884.

P.-A. : Eaton (1884) ; Thibault (1971) ; Vinçon \& Thomas (1987). Ar. : l'Arize au Mas d'Azil (325 m).

\section{Famille des Polymitarcyidae}

Genre Ephoron Williamson, 1802

55. E. virgo (Olivier, 1791).

H.-G. : Eaton (1883), s. n. Polymitarcys virgo. (Olivier), confirmant N. Joly \& E. Joly (1878) et les citations antérieures de l'un ou des deux auteurs, confuses et redondantes, depuis 1871 ; la Louge à St-Hilaire $(200 \mathrm{~m})$. T.: le Tarn à Albi $(175 \mathrm{~m})$ (N. Giani leg.) ; le Gijou à Vabre $(370 \mathrm{~m})$ (A. Raynaud leg.). Av.: la Vayre à Quins (450 m) (J. Guitard leg).

\section{Famille des Ephemeridae} Genre Ephemera Linné, 1746

56. E. danica Müller, 1764.

P.-A. : Thibault (1971), Vinçon \& Thomas (1987). H.-P: : le Nistos à Bonrepayre (490 m). H.-G. : la Garonne en amont de St-Gaudens $(365 \mathrm{~m})$; le Tounis près de Cazères $(240 \mathrm{~m})$ (J.Y. Pujol leg.). Ar. : le Lez à Moulis (430 m) (C. Berthélemy leg.) ; le Volp, de sa perte $(460 \mathrm{~m})$ à l'amont de Mérigon $(310 \mathrm{~m})$; la cascade en forêt de Ste-Croix (320 m) (C. Berthélemy leg.) ; l'Arize au Mas d'Azil (325 m). Au. : l'Aude en aval de Puyvalador $(1370 \mathrm{~m})$. Av. : le Lot à St-Geniez (425 m) (A.T \& C. Laur leg.). Ca. : le ruisseau de Monreysse à $530 \mathrm{~m}$, près d'Aurillac (G. Gazagnes leg.).

57. E. glaucops Pictet, 1843.

H.-G. : Eaton (1883). Espèce non citée à nouveau de la région, à ma connaissance.

58. E. lineata Eaton, 1870.

H.-G. : la Garonne à Portet $(145 \mathrm{~m})$ et à Toulouse $(130 \mathrm{~m})$ (Thomas et al. 1992). Ar. : le Volp au pont du Luquet $(240 \mathrm{~m})$ (C. Berthélemy \& A.T. leg.) ; l'Arize au Mas d'Azil (325 m).

59. E. vulgata Linné, 1746.

H.-G. : le Canal du Midi à Toulouse $(125 \mathrm{~m})$ et l'Hers à Lasbordes (140 m) (R. Despax leg.). Ar. : le Volp à Montardit (430 m) (C. Berthélemy. \& A. T. leg.). 


\section{Famille des Potamanthidae}

Genre Potamanthus Pictet, 1843

60. P. luteus (Linné, 1789).

P.-A. : Bertrand \& Verrier (1949b). H.-G. : Eaton (1884). Au. : Eaton (ibid.) ; l'Aude à Pomas ( $150 \mathrm{~m})$ et en amont de Carcassonne (115 m). T. \& G. : la Garonne à Verdun-sur-Garonne $(105 \mathrm{~m})$. Av. : le Lot entre StGeniez (425 m) et Boisse-Penchot (175 m) (A.T. \& C. Laur leg.). Loz. : le Lot à Montjézieu (535 m).

\section{Discussion et conclusion}

Sept espèces sur les 60 signalées ci-dessus ont été décrites à partir de matériel provenant exclusivement du Sud-Ouest de la France : une par E. Joly (Neoephemera maxima) et six par Thomas ou Thomas \& Gazagnes. En fait, $N$. maxima a été citée depuis de Pologne (Jazdzewska 1975), et Ecdyonürus angelieri a été trouvée en Espagne du Nord-Ouest. Peuvent donc être actuellement considérées comme endémiques des Pyrénées (et probablement de leur seul versant Nord) : Baetis catharus, B. nicolae, Rhithrogena kimminsi, $R$. strenua et Habroleptoides berthelemyi.

Trois autres espèces ont été érigées à partir de matériel d'une plus large aire de répartition, mais dont la Terra typica est le Sud-Ouest de la France :

- soit par désignation dans la description originale, cas d'Ecdyonurus macani Thomas \& Sowa, trouvée aussi en Pologne, puis plus tard en Tchécoslovaquie (Krno 1981);

- soit par désignation ultérieure (Kimmins 1960) de lectotypes récoltés dans le Sud-Ouest de la France,' dans le cas de Procloeon pulchrum (Eaton) et d'Epeorus torrentium Eaton.

Parmi les actions anthropiques les plus à craindre pour la survie de la faune de la région, il faut mentionner le grand nombre de retenues (plusieurs centaines), de types différents et de capacité très variable.

Les innombrables prélèvements d'eau pour l'irrigation, autorisés ou non, abaissent -lors des étiages extrêmes- le module à des valeurs critiques : le marnage provoque alors l'émersion répétée d'une grande partie du lit. Ce phénomène est particulièrement marqué pendant plusieurs mois par an sur le dernier tronçon lotique (épipotamal) de la Garonne près de Toulouse, le module pouvant être inférieur à $50 \mathrm{~m} 3 / \mathrm{s}$ à l'étiage pour une largeur de lit supérieure à $100 \mathrm{~m}$. Néanmoins à l'heure actuelle, l'aspect le plus préoccupant -au moment où les rejets urbains toxiques sont l'objet d'une surveillance accrue- paraît être l'usage, abusif et incontrôlé, des engrais et des pesticides/herbicides etc. en zone rurale.
En dessous d'une altitude de $500 \mathrm{~m}$, la richesse spécifique des peuplements d'insectes aquatiques de l'hyporhithral et de l'épipotamal est en forte régression depuis 25 ans : ainsi, Rhithrogena germanica, Ecdyonurus macani, Ephemera glaucops et E. vulgata, devenues très rares, sont gravement menacées. Pour les mêmes raisons, Isonychia ignota, Neoephemera maxima, Brachycercus harisella et Ephemera glaucops, non retrouvées depuis plus d'un siècle, à ma connaissance, ainsi que Prosopistoma punctifrons depuis plus de soixante ans, ont probablement déjà disparu de la région à l'heure actuelle.

Le nombre d'espèces d'Ephémères qui restent à citer du Sud-Ouest de la France est probablement compris entre 20 et 30 , en tenant compte des formes rares non encore signalées et des espèces déjà connues de la région, mais qui posent actuellement soit un problème de nomenclature, soit un problème de caractérisation, soit les deux à la fois. Ces espèces appartiennent essentiellement aux genres Electrogena, Rhithrogena et Habrophlebia. Le nombre total d'espèces peut ainsi être estimé entre 80 et 90 .

\section{Remerciements}

J'ai plaisir à remercier plusieurs de mes collègues pour avoir effectué des récoltes à mon intention : Jeanine Guitard, Narcisse Giani, Joël Moubayed-Breil, Albert Raynaud, Max Thibault et JeanNoël Tourenq, ainsi que Patrick McCafferty, Lafayette, Indiana, pour sa traduction du résumé.

\section{Travaux cités}

Berthélemy C. \& Thomas A. 1967.- Note taxonomique sur Epeorus torrentium Eaton, 1881 et $E$. assimilis Eaton, 1885 (Ephemeroptera, Heptageniidae). Annls Limnol., 3 (1) : 65-74.

Bertrand H. \& Verrier M.L. 1949a.- Contribution à la biogéographie des Ephéméroptères des Pyrénées. Bull. biol.Fr. Belg., 83 (1) : 1-24.

Bertrand H. \& Verrier M.L. 1949b.- Nouvelles stations françaises d'Ephéméroptères (3' note). Bull. Soc. ent. Fr., 54:121-124.

Bertrand H. \& Verrier M.L. 1950a.-Contribution à l'étude de la faune des eaux douces de la région orientale des Pyrénées. Vie Milieu, 1 (2) : 217-234.

Bertrand H. \& Verrier M.L. 1950b.-Contribution à l'étude de la faune des eaux douces de la région orientale des Pyrénées (Deuxième partie). Vie Milieu, 1 (4) : 449-459.

Despax R. 1927.- Nouvelles stations françaises de Siphlurus lacustris Eaton (Ephemeridae). Bull. Soc. ent. Fr., 1927 (n9) : 151-152.

Despax (R.). 1949.- Addition à la faune des Ephéméroptères de France : Rhithrogena haarupi dans les Pyrénées. Bull. Soc. Hist. nat. Toulouse, 84 (3-4) : 145-146.

Eaton A.E. 1883-1888.- A revisional monograph of recent Ephemeridae or Mayflies. Trans. Linn. Soc., 3 (Zoology) : 1-352 + 65 pl.

Gagneur J., Thomas A.G.B. \& Vitte B. 1985. - Première citation d'une espèce du genre Paraleptophlebia en Afrique du Nord: $P$. cincta (Retzius, 1783) et son écologie (Ephemeroptera, Leptophlebiidae). Bull. Soc. Hist. nat. Toulouse, 121 : 141-143. 
Jazdzewska T. 1975.- Neoephemera maxima (Joly, 1870) (Ephemeroptera, Neoephemeridae) in Poland. Polskie Pismo ent., 45 : 227-238.

Joly E. 1870.- Contributions pour servir à l'histoire naturelle des Ephémérines. Bull. Soc. Hist. nat. Toulouse, 4 : 142-151 + 1 pl.

Joly E. 1871.- Note sur le prétendu crustacé dont Latreille a fait le genre Prosopistoma. Mem. Soc. Sci. nat., Cherbourg, 16 : 329 336.

Joly E. 1872. - Contributions pour servir à l'histoire naturelle des Ephémérines. $\mathrm{N}^{\circ} 2$. Description de la nymphe d'une Ephémérine à très longues soies caudales Palingenia roeselii, Nob. observée pour la première fois sur les bords de la Garonne, au mois de septembre 1868. Mem. Soc. natn. Sci. nat. Cherbourg, 16:1-16 $+1 \mathrm{pl}$.

Joly E. 1873. - Texte d'une communication présentée par Mr Müller dans la rubrique «Proceedings of the Entomological Society», June 2, 1873, The Zoologist : pp 3655-3657.

Joly N. \& Joly E. 1872.— Etudes sur le prétendu Crustacé au sujet duquel Latreille a créé le genre Prosopistoma et qui n'est autre chose qu'un véritable insecte Hexapode. Annls. Sci. nat., Zool., 5e série, $16: 1-16+1 \mathrm{pl}$.

Joly N. \& Joly E. 1875.- Nouvelles recherches tendant à établir que le prétendu Crustacé décrit par Latreille sous le nom de Prosopistoma est un véritable insecte de la tribu des Ephémérines. Rev. Sci. nat., 4 (juin) : $1-15+1$ pl.

Joly N. \& Joly E. 1878.- Etudes sur les métamorphoses et l'embryogénie des Ephémérines, et spécialement sur celles de la $\mathrm{Pa}$ lingenia virgo. Ass. Fr. Avancemt Sci., Congrès de Paris, 10 section, Zoologie : 717-730.

Kimmins D.E. 1960. - The African species of the genus Cheumatopsyche (Trichoptera, Hydropsychidae), and the Ephemeroptera types of species described by A.E. Eaton, R. McLachlan, and F. Walker. Bull. Br. Mus. (Nat. Hist.), Entomol., 9 (4) : 255-318.

Krno I. 1981.-Ecdyonurus macani Thomas et Sowa, novy druh podeniek (Ephemeroptera) pre faunu Ceskoslovenska. Biologia (Brätislava), $36(5): 389-390$

Lavandier P. \& Dumas J. 1971.— Microrépartition de quelques espèces d'invertébrés benthiques dans des ruisseaux des Pyrénées Centrales. Annls Limnol., 7(1) : 7-23.

McCafferty W.P. \& Waltz R.D. 1990.- Revisionary synopsis of the Baetidae (Ephemeroptera) of North and Middle America. Trans. Am. Ent. Soc., 116 (4) : 769-799.

McCafferty W.P. \& Waltz R.D. 1995.- Labiobaetis (Ephemeroptera : Baetidae) : new status, new North American species, and related new genus. Ent. News, 106 (1) : 19-28.

Malzacher P. 1986. - Diagnostik, Verbreitung und Biologie der europäischen Caenis-Arten (Ephemeroptera : Caenidae). Stuttg. Beitr. Naturk, Ser. A, $n^{\circ} 387: 1-41$.

Müller-Liebenau I. 1969.- Revision des europäischen Arten des Gattung Baetis Leach, 1815 (Insecta, Ephemeroptera). Gewäss.-Abwäss., 48/49: 1-214.

Müller-Liebenau I. 1974.- Baetidae aus Südfrankreich, Spanien und Portugal (Insecta, Ephemeroptera). Gewäss.-Abwäss., 53/54: $4-42$

Sartori M. 1990.- First record of the genus Brachycercus Curtis, 1834 in the Iberian Peninsula (Ephemeroptera, Caenidae). Eos, $66(2): 229-235$.

Sartori M. \& Thomas A.G.B. 1991.- Contribution to the systematics of Baetis muticus (L.) and allied species from South Western palearctic region (Ephemeroptera; Baetidae). In J. AlbaTercedor \& A. Sanchez-Ortega (eds) : Overview and strategies of Ephemeroptera and Plecoptera (pp 223-233). Sandhill Crane Press, Gainesville.
Sowa R. 1971.- Note sur quelques Rhithrogena Eaton de la collection Esben-Petersen et la redescription de Rhithrogena germanica Eaton (Ephemeroptera, Heptageniidae). Bull. Acad. pol. Sci. (Sér. Sci. biol.), 19 (7-8) : 485-492.

Tabacchi E. 1987.- Thraulus bellus Eaton, 1881 : nouvelle station de récolte et notes préliminaires sur son habitat (Ephemeroptera, Leptophlebiidae). Bull. Soc. Hist. nat. Toulouse, $123: 81-84$.

Thibault M. 1971.- Le développement des Ephéméroptères d'un ruisseau à truites des Pyrénées-Atlantiques, le Lissuraga. Annls Limnol., 7(1) : 53-120.

Thomas A. 1968a.- Sur la taxonomie de quelques espèces d'Ecdyonurus du Sud-Ouest de la France (Ephemeroptera). Annls Limnol., 4 (1) : 51-71.

Thomas A. 1968b.- Habrophlebia (Habroleptoides) berthelemyi n. sp. des Pyrénées (Ephemeroptera, Leptophlebiidae). Annls Limnol., 4 (2) : 219-224.

Thomas A. 1970a.- Taxonomie et répartition des Ephéméroptères et de quelques Diptères aquatiques (Tipuloidea et Psychodidae) des Pyrénées. Thèse Spécialité, Univ. Paul Sabatier, Toulouse : $105 \mathrm{p}$.

Thomas A. 1970b.- Sur la taxonomie de deux Rhithrogena des Pyrénées (Ephemeroptera, Heptageniidae). Annls Limnol., 6 (3) : 305-315.

Thomas A.G.B. 1975.- Ephéméroptères du Sud-Ouest de la France. I.- Migrations d'imagos à haute altitude. Annls Limnol., 11 (1) : 47-66.

Thomas A. 1981.-Travaux sur la taxonomie, la biologie et l'écologie d'insectes torrenticoles du Sud-Ouest de la France (Ephéméroptères et Diptères : Dixidae, Cecidomyiidae, Rhagionidae et Athericidae), avec quelques exemples de perturbations par l'homme. Thèse Doctorat d'Etat, Univ. Paul Sabatier Toulouse, $\mathrm{n}^{\circ} 988: 330 \mathrm{p}$.

Thomas A.G.B. 1986.- Ephéméroptères du Sud-Ouest de la France. IV. Baetis catharus n. sp. des Pyrénées (Baetidae). Bull. Soc. Hist. nat. Toulouse, 122 : 175-179.

Thomas A. 1995.- Macroinvertebrates limit communities in headwaters at the highest altitudes in the Pyrenees of Southwestern France. 43rd Annual Meeting, Keystone Resort, Colorado ; Bulletin NABS $12(1): 187$

Thomas A.G.B. \& Gazagnes G. 1982.- Ephéméroptères du SudOuest de la France. II. Rhithrogena strenua $\mathrm{n}$. $\mathrm{sp}$. des Pyrénées (Heptageniidae). Bull. Soc. Hist. nat. Toulouse, $118: 291-295$.

Thomas A.G.B. \& Gazagnes G. 1983.- Ephéméroptères du SudOuest de la France. III. Baetis nicolae n. sp. des Pyrénées (Baetidae). Bull. Soc. Hist. nat. Toulouse, 119: 71-74.

Thomas A.G.B., Prévot R. \& Vinçon G. 1986.- Deux Ephéméroptères nouveaux pour la faune de France : Baetis pentaphlebodes Ujhelyi, 1966 (Baetidae) et Caenis beskidensis Sowa, 1973 (Caenidae). Bull. Soc. Hist. nat. Toulouse, $122: 179$.

Thomas A. \& Sowa R. 1970.-Ecdyonurus macani n. sp., espèce européenne voisine d'E. torrentis Kimmins (Ephemeroptera, Heptageniidae). Annls Limnol., 6 (1) : 75-85.

Thomas A. \& Thomas N. 1987.- Diptères torrenticoles peu connus. XI. Les Athericidae du Sud de la France (La dérive des larves d'A. ibis en haute montagne) (Brachycera, Orthorrhapha). Bull. Soc. Hist. nat. Toulouse, $123: 89-94$.

Thomas A., Thomas N. \& Khatori M. 1992.- Ephemeroptera as bioindicators compared to other lotic insects over long term evolution in the Garonne River near Toulouse (South Western France). 7th Internat. Conf. Ephemeroptera, Orono, Maine, U S A.

Thomas A.G.B. \& Vitte B. 1988.-Compléments et corrections à la faune des Ephéméroptères d' Afrique du Nord. 1. Le genre Choroterpes Eaton, sensu stricto (Ephemeroptera). Annls Limnol., $24(1): 61-65$. 
Thomas A.G.B., Vitte B. \& Soldan T. 1987.-Rhithrogena ryszardi n. sp., Ephéméroptère nouveau du Moyen Atlas (Maroc) et redescription de Rh. soteria Navas, 1917 (Heptageniidae). Annls Limnol., 23 (3) : 169-177.

Vayssière A. 1881.-Etude sur l'état parfait du Prosopistoma punctifrons. Annls Sci. nat., Zool., 6 série, 11 (1): 1-16+1 pl. et Bibltque Ec. ht. Etud., Paris Sect. Sci. nat., 22 (1): 1-16+1 pl.

Vayssière A. 1882.- Recherches sur l'organisation des Ephémérines. Annls Sci. nat., Zool., 6' série, 13 (1): 1-137+11 pl.
Vinçon G. \& Thomas A.G.B. 1987.- Etude hydrobiologique de la vallée d'Ossau (Pyrénées-Atlantiques). I. Répartition et écologie des Ephéméroptères. Annls Limnol., 23 (2) : 95-113.

Waltz R.D., McCafferty W.P. \& Thomas A. 1994.- Systematics of Alainites n. gen., Diphetor, Indobaetis, Nigrobaetis n. stat., and Takobia n. stat. (Ephemeroptera, Baetidae). Bull. Soc. Hist. nat. Toulouse, $130:$ 33-36. 